\title{
Preoperative Serum Prostate-Specific Antigen Level is a Predictive Factor for Biochemical Recurrence in High Risk Prostate Cancer after Radical Prostatectomy
}

\author{
Tetsuya Shindo $^{1}$, Kohei Hashimoto ${ }^{2}$, FumimasaFukuta ${ }^{3}$, Naoya Masumori ${ }^{4}$ \\ ${ }^{1,2,3,4}$ Department of Urology, Sapporo Medical University School of Medicine, Sapporo, Japan
}

\begin{abstract}
Objective: To evaluate preoperative predictive factor for biochemical recurrence (BCR) after radical prostatectomy $(\mathrm{RP})$ for high risk prostate cancer $(\mathrm{PCa})$.

Methods: We retrospectively evaluated 96 patients with clinically localized or minimally invasive high risk $P C a$ who underwent RP. High risk PCa was defined by D'Amico's criteria prostate-specific antigen (PSA)> 20 $n g / m l$ or clinical $T 2 c / 3$ or a biopsy Gleason Score $\geq 8$.The log-rank test and Cox proportional hazards analysis were used to evaluate predictive factors.

Results: The follow-up periods ranged from 1-111 months (median 43.1 months). Twenty-four of 96 (25\%) patients had BCR after RP but only one patient (1\%) died of cancer during the follow -up period. The five-year $B C R$-free survival rate was $68.4 \%$ for all patients. A preoperative serum PSA level of more than $20 \mathrm{ng} / \mathrm{ml} \mathrm{was} \mathrm{a}$ risk factor for BCR ( $p=0.008$, HR3.632 95\%CI 1.402-9.409, Cox proportional hazards analysis) and patients having multiple risk factors had a higher risk for BCR than patients with a single risk factor ( $p=0.003$, log-rank test).
\end{abstract}

Conclusion: A serum PSA level of more than $20 \mathrm{ng} / \mathrm{ml}$ is a risk factor for BCR in high risk PCa after RP. Patients having multiple risk factors have a high risk for BCR.

Keywords: High risk prostate cancer, Biochemical recurrence, Radical prostatectomy, Risk factor

Abbreviations

BCR: biochemical recurrence

$R P$ : radical prostatectomy

PCa: prostate cancer

ADT: androgen deprivation therapy

GS: Gleason score

PSA: prostate specific antigen

$R R P$ : retropubic radical prostatectomy

LRP: laparoscopic radical prostatectomy

RALP: robot-assisted laparoscopic radical prostatectomy

PLND: pelvic lymph node dissection, ECE: extracapsular extension

PSAD: prostate specific antigen density

TRUS: transrectal ultrasound

\section{INTRODUCTION}

Radical prostatectomy (RP) and radiation therapy (RT), with or without androgen deprivation therapy (ADT), are standard therapies again storgan-confined prostate cancer (PCa). Even after standard treatment, some patients having PCa unfortunately experience biochemical recurrence (BCR) and require additional therapy such as salvage RT or androgen deprivation therapy (ADT). Thus, BCR can seriously affect a patient's life after RP. On the other hand, some patients achieve long disease-free survival with only RP even with high risk cancer defined as clinical T stage T2c/T3 ora biopsy Gleason $\operatorname{score}(\mathrm{GS}) \geq 8$, and serum prostate-specific antigen(PSA) level of $>20 \mathrm{ng} / \mathrm{ml}$ by D'Amico et al.[1]. Meng et al. reported that $26 \%$ of PCa patients met D'Amico's high risk criteria in the CaPSURE population [2]. Yamamoto et al. reported that RP for localized high risk PCa provided a 
cure in approximately 50\% of high risk PCa patients [3].Although several reports have discussed predictive factors for BCR after RP, the definitive preoperative predictive factors in high risk PCa are not clear. Preoperative prediction of BCR is essential and it may enable appropriate treatment selection such as RP or RT+ADT for high risk PCa patients.

Thus, we focused on RP for high risk PCa and performed a single center retrospective study to evaluate the preoperative parameters for BCR after RP in high risk PCa patients.

\section{MATerials AND Methods}

We retrospectively evaluated 96 patients with clinically localized or minimally invasive (cT3)high risk PCa who underwent RP at our institution from January 2005 through July 2014.D'Amico's criteria were used for definition of high risk PCa (PSA more than 20ng/ml, cT2c/T3, GS 8 or more) [1]. All cases of PCa were staged according to the American Joint Committee on Cancer Staging Manual [4]. All patients underwent computed tomography and bone scintigraphy before RP to confirm that they did not have any radio graphically detectable metastatic disease. Patients who received neoadjuvant ADT or who's cancer diagnosed by transurethral resection of the prostate were excluded. BCR was defined as a postoperative PSA level of more than $0.2 \mathrm{ng} / \mathrm{ml}$. Patients who did not achieve a postoperative PSA nadir of less than $0.2 \mathrm{ng} / \mathrm{ml}$ were considered to have BCR at the time of RP. Radical surgery was performed using several methods, including retro pubic RP (RRP), laparoscopic RP (LRP) and robot-assisted laparoscopic RP (RALP). Extended pelvic lymph node dissection (PLND) was not performed during the study period and only limited PLND was performed for all patients. All specimens were pathologically evaluated by an experienced pathologist in our hospital. Statistical analysis was performed using the log-rank test for intergroup BCR-free survival comparisons. Cox proportional hazards analysis was performed to evaluate predictive factors for BCR. All values were considered to be statistically significant at $\mathrm{P}<0.05$. Statistical analyses were performed using SPSS version 16.0. This study was approved by the institutional review board in our hospital (No. 272-178), which was performed in accordance with the principles outlined in the Declaration of Helsinki.

\section{RESULTS}

Table1. Patient's characteristics

\begin{tabular}{|l|l|}
\hline & $\mathrm{N}=96$ \\
\hline Age median(range) & $66(51-75)$ \\
\hline PSA (ng/ml) Median (range) & $9.19(2.55-46.78)$ \\
\hline PSAD $\mathrm{ml}^{-1} \mathrm{~cm}^{-2}$ & $0.29(0.09-2.18)$ \\
\hline Prostate volume (cc) & $27.65(11.2-113)$ \\
\hline Median (range) & \\
\hline cT stage & $35(36.5 \%)$ \\
1c & $32(33.3 \%)$ \\
2a & $8(8.3 \%)$ \\
2b & $17(17.7 \%)$ \\
\hline 2c & $4(4.2 \%)$ \\
\hline 3a & $12(5-20)$ \\
\hline Prostate biopsy core number median (range) & \\
\hline Gleason Sum & $7(7.3 \%)$ \\
\hline 6 & $19(19.4 \%)$ \\
7 & $52(54.1 \%)$ \\
8 & $18(18.8 \%)$ \\
\hline 9 & \\
\hline Surgery & $70(72.9 \%)$ \\
RRP & $15(15.6 \%)$ \\
LRP & $11(11.5 \%)$ \\
\hline RALP & \\
\hline Nerve Sparing & $64(66.7 \%)$ \\
None & $27(28.1 \%)$ \\
\hline Unilateral & $5(5.2 \%)$ \\
\hline Bilateral & $43.1(1-111)$ \\
\hline Follow up period (months) median (range) & $24(25 \%)$ \\
\hline BCR & \\
\hline
\end{tabular}


Preoperative serum prostate-specific antigen level is a predictive factor for biochemical recurrence in high risk prostate cancer after radical prostatectomy

Table 2.Pathological outcome of radical prostatectomy specimen

\begin{tabular}{|l|l|}
\hline & $\mathrm{N}=96$ \\
\hline pT stage & \\
2a & $24(25 \%)$ \\
2b & $14(14.6 \%)$ \\
2c & $25(26 \%)$ \\
3a & $20(20.8 \%)$ \\
3b & $13(13.5 \%)$ \\
\hline pN stage & \\
N0 & $91(94.8 \%)$ \\
N1 & $5(5.2 \%)$ \\
\hline Resection margin (RM) & \\
RM+ & $37(38.5 \%)$ \\
RM- & $57(59.4 \%)$ \\
RMx & $2(2.1 \%)$ \\
\hline Capsular invasion(Cap) & \\
Cap + & $29(30.2 \%)$ \\
Cap- & $63(65.6 \%)$ \\
Cap x & $4(4.2 \%)$ \\
\hline Gleason Sum & \\
6 & $4(4.2 \%)$ \\
7 & $44(45.8 \%)$ \\
8 & $22(22.9 \%)$ \\
9 & $26(27.1 \%)$ \\
\hline Retrieved lymph nodes number (median) & $0-23(6)$ \\
\hline
\end{tabular}

Table3. Multivariate analysis of predictive factor for PSA failure after RP (Cox proportional hazard analysis)

\begin{tabular}{|l|l|l|}
\hline Factor & HR (95\%CI) & P-value \\
\hline cT stage cT2c or more & $0.694(0.231-2.088)$ & 0.516 \\
\hline Gleason Score 8 or more at prostate biopsy & $2.070(0.714-6.004)$ & 0.181 \\
\hline PSA level more than 20ng/ml & $3.632(1.402-9.409)$ & 0.008 \\
\hline
\end{tabular}

The characteristics of the patients are shown in Table 1. Twenty-four patients $(24 / 96,25 \%)$ had BCR but only one $(1 / 96,1 \%)$ died from cancer during the follow-up period. Seven of 96 (7.3\%) patients had BCR without achieving PSA nadir less than $0.2 \mathrm{ng} / \mathrm{ml}$ after RP. Ten of 96 (10.4\%) patients underwent salvage radiation therapy after BCR. Because we started RALP from 2013 and mainly employed it for low/intermediate risk PCa patients, only 11 patients were treated by the method. The pathological outcomes are shown in Table 2. Although all patients received RP with at least limited lymph node dissection, 2 patients had no lymph node in final pathological report. Though only 4 patients $(4 / 96,4.2 \%)$ were evaluated to have cT3a preoperatively, 33 (33/96,34.3\%) had pT3a or more in the final pathologic evaluation. The distribution of the risk factors in the cohort is shown in Figure1.

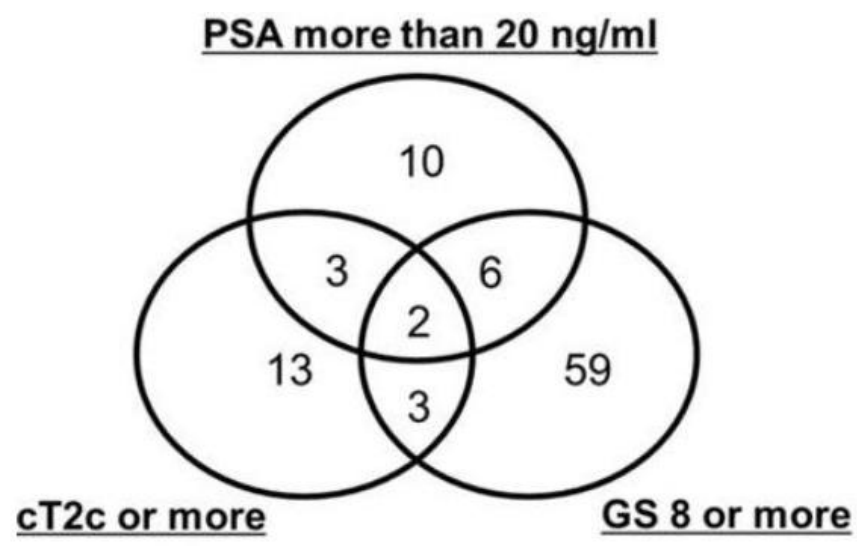

Figure1.The distribution of high risk PCa in the cohort 
Ten patients had high risk PCa based only on the PSA level, 13 based on the cT stage, and 59 based on the GS. Fourteen patients had multiple risk factors according to D'Amico's criteria. Only 2 patients had all 3 risk factors. The BCR-free survival rate is shown in Figure 2. The five-year BCR-free survival rate for all patients was $68.4 \%$. The BCR-free survival rates according to risk factors are presented in Figure 3. The GS determined by prostate biopsy (Figure 3a) and the cT stage (Figure 3b) were not able to predict BCR (log-rank test, $\mathrm{p}=0.699$, $\mathrm{p}=0.441$ ). On the other hand, the preoperative PSA level showed a significant difference for BCR- free survival (Figure 3c, log-rank test, $p=0.022$ ) and patients having multiple risk factors were highly likely to have BCR (Figure 3d, log-rank test, $\mathrm{p}=0.003$ ). Table 3 shows that a serum PSA level of more than $20 \mathrm{ng} / \mathrm{ml}$ was a predictive factor for $\mathrm{BCR}$ in multivariate analysis ( $\mathrm{p}=0.008$, Cox proportional hazard analysis).

The concordance between the GS by determined by prostate biopsy and the final pathology is shown in Figure 4a. Although 73\% of the cohort was diagnosed as having GS 8 or higher in prostate biopsy, only $50 \%$ had GS 8 or more in the final pathology. On the other hand, while $21.9 \%$ were diagnosed as having clinical stage T2c or more at the preoperative evaluation, $60.3 \%$ did in the final pathology.

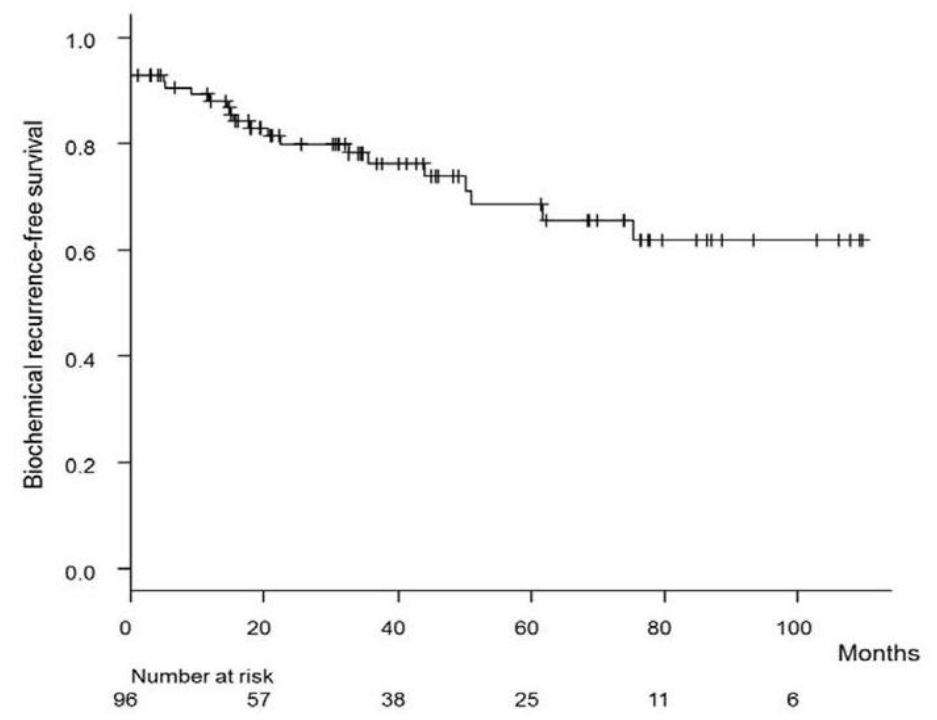

Figure2.Biochemical recurrence free survival of the cohort
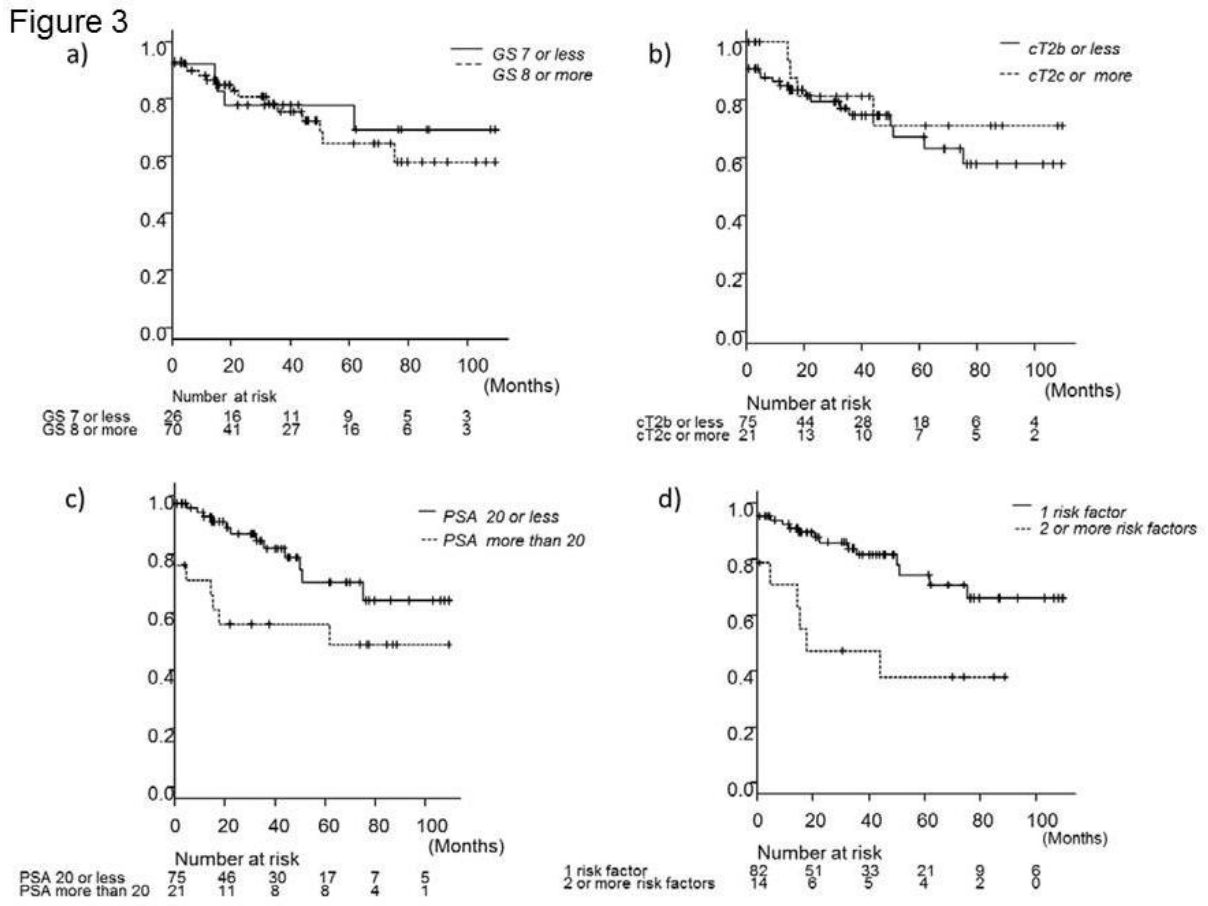

Figure3.Biochemical recurrence free survival rate according to risk factors (Log-rank test). 


\section{DISCUSSION}

It is reported that RT with ADT is superior to RT only for high risk PCa [5]. However, it is not clear whether RT with ADT is superior to RP for high risk PCa. Yamamoto et al. reported that RP for high risk PCa resulted in 50\% cancer-specific survival in long-term follow up (median: 8.1 years)[3]. Thus, high risk PCa includes cancer with heterogeneous biological behavior and some of them might be good candidate's forcure by RP alone. Therefore, preoperative predictors of clinical outcomes for the patients are essential to determine the optimal treatment strategy. Abdollah et al. reported the long-term outcomes of RALP for high risk PCa and classified the biochemical recurrence risk by the biopsy GS and preoperative PSA leve 1[6]. According to the study, the 10-year BCR-free survival rate was $26.2 \%$ in patients with PSA levels of $10 \mathrm{ng} / \mathrm{ml}$ or more and GS8 or more. On the other hand, that of patients with PSA $10 \mathrm{ng} / \mathrm{ml}$ or less and GS 7 or less the BCR survival rate was $69.8 \%$.Although D'Amico's criteria include three factors to determine high risk PCa, which one best reflects the tumor biology and oncological outcome is not clear. In the present study, the biopsy GS and cT stage did not predict outcomes. There was a discrepancy between the biopsy GS and final pathology (Figure 4a). Although $73 \%$ of the cohort had GS 8 or more at biopsy, only $50 \%$ had such a GS in the final pathology. The number of prostate biopsy core in the cohort contributed to the concordance of Gleason score at biopsy and final pathology. Although only 1 patient received 5-core biopsy, 21 of 96 (21.9\%) patients received less than 10 cores. Previous study reported that 10 or more cores biopsy may improve concordance of biopsy and RP GS [7]. The discrepancy of the GS may result in its failure to be a predictive factor. Van Poppel et al. reported that one-third of patients with GS8-10 at biopsy had GS 7 or less in the final pathologic report [8].Thus, overestimation of the GS may occur in biopsy specimens. For the cT stage as well, the concordance was not good enough to predict the outcome (Figure 4b). Although only $4.2 \%$ of the cohort had cT3a, 34.3\% had pT3a or more in the final pathology. Thus, extracapsular extension (ECE) and seminal vesicle invasion could not be precisely detected by preoperative evaluation in the present study. Hardeman et al reported an assessment of the ECE of PCa by transrectal ultrasound (TRUS) and the sensitivity, specificity and accuracy were 54\%, 58\% and 56\%, respectively [9]. The detection of cancers having extracapsular extension (ECE) might be improved by using high power field MRI. Three tesla MRI (3T-MRI) is reported to have higher sensitivity and specificity (66.7\% and 100\%) for detection of ECE in PCa than 1.5T-MRI[10].

Not only under staging, but also over staging, may occur in the evaluation of the clinical T stage. Ward et al. reported that $13 \sim 27 \%$ of cT3a PCa patients were overestimated and had pT2 disease [11]. In the cohort, 37 of 96 (38.5\%) patients had positive surgical margin in RP. Positive surgical margin was a significant risk factor for BCR ( $\mathrm{p}=0.0164$, Log-rank test). The effect of positive surgical margins may affect uncertainty of $\mathrm{T}$ stage to predict $\mathrm{BCR}$. Thus, clinical staging may not be reliable as a preoperative predictive factor for BCR after RP.

When comparing PSA level and postoperative final pathological T stage and GS in final pathology together in multivariate analysis as a predictive factor for BCR, GS 8 or higher $(\mathrm{P}=0.004$, cox proportional hazard model) and PSA level $20 \mathrm{ng} / \mathrm{ml}$ or more $(\mathrm{P}=0.009$, cox proportional hazard model) were statistically significant factors. However, assessment of BCR risk in preoperative factor is crucial for patients.

Our results suggested that the serum PSA level was the most significant predictor. Yamamoto et al. evaluated the impacts of three factors on BCR in high risk PCa. Among 106 high risk prostate cancer patients, the 10-year BCR-free survival rates were $69.1 \%$ for $\mathrm{GS} \geq 8$ alone, $56.1 \%$ for cT3 alone and $47.8 \%$ for PSA of more than $20 \mathrm{ng} / \mathrm{ml}$ alone, respectively. Thus, a high PSA level was associated with the worst BCR-free survival in high risk PCa patients after RP [3]. The PSA density (PSAD) is also reported to be a useful predictive factor for BCR after RP for high risk PCa[12], [13].However, the cutoff points vary among reports because they were determined in specific limited cohorts. Koie et al. reported a cutoff point for BCR after RP in high risk PCa of $0.468 \mathrm{ng} / \mathrm{ml} / \mathrm{cm} 3[12]$ and Hamada et al as $0.4 \mathrm{ng} / \mathrm{ml} / \mathrm{cm} 3$ [13]. Thus, it is difficult to select the optimal cut off point. Furthermore, prostate volume evaluated by TRUS is reported to result in both over- and underestimation [14] and might influence the PSAD. It is also reported that MRI may be more accurate for estimating prostate volume than TRUS [15]. However, extremely high PSAD such as that over $1.0 \mathrm{ng} / \mathrm{ml} / \mathrm{cm} 3$ was reported to 
involve a high risk for BCR after RP[16]. Thus, it may be reasonable for PCa patients having a PSA just below 20ng/ml and prostate volume of $20 \mathrm{cc}$ who have PSAD of almost $1.0 \mathrm{ng} / \mathrm{ml} / \mathrm{cm} 3$, to be considered at high risk according to the PSA Deven though it does not satisfy D'Amico's criteria.

(\%)

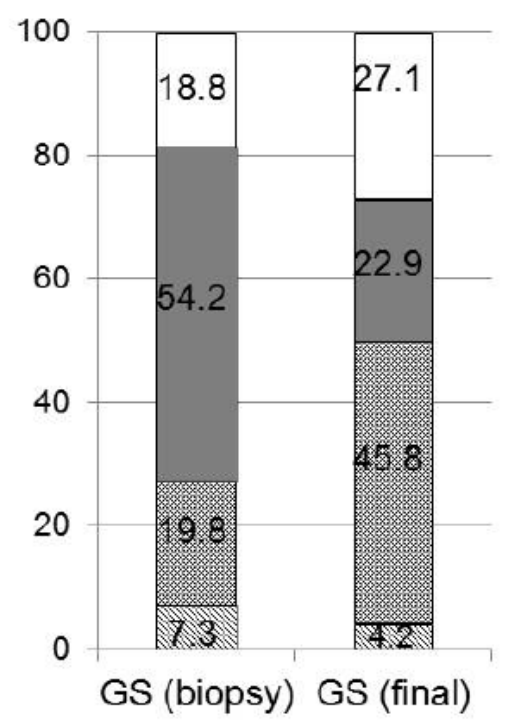

(\%)

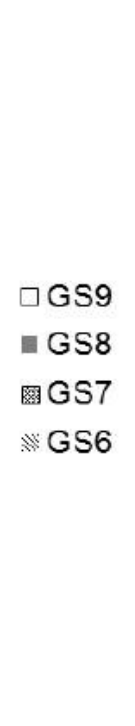

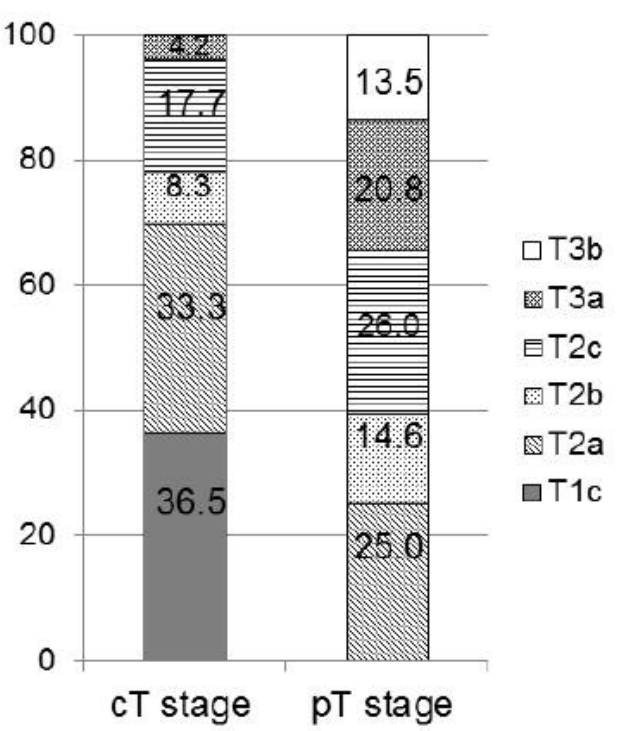

Figure4. Concordance for a) preoperative and final Gleason score and b) clinical and pathological T stage

High risk PCa patients with multiple risk factors, such as GS 8 or more and a PSA level of more than $20 \mathrm{ng} / \mathrm{ml}$ had an extremely high risk for BCR (Figure 3d). Previous reports showed the same results[3],[12].According to Aoun F et al., higher PSA was a preoperative predictive factor for BCR in negative surgical margin T2 prostate cancer patients[17]. Therefore, we must be aware of the risk when considering RP for such patients.

There are several limitations in the present study. We did not perform extended PLND, which might have therapeutic potential for cancer control [18] and the number of patients who had BCR was relatively small. In addition, this was a retrospective study and most of the patients were treated by RRP, which was mainly done by RALP, recently. Suardi et al. reported that RALP may reduce positive surgical margin in high risk prostate cancer patients [19]. Considering our cohort's complexity of surgical technique, our conclusion may not simply apply in RALP era.

\section{Conclusions}

A serum PSA level more than $20 \mathrm{ng} / \mathrm{ml}$ may be a predictive factor for BCR in high risk PCa after RP. We should be aware of the high risk of BCR after RP in patients having multiple risk factors defined by D'Amico's criteria.

\section{REFERENCES}

[1] D'Amico AV, Whittington R, Malkowicz SB, et al. Biochemical outcome after radical prostatectomy, external beam radiation therapy, or interstitial radiation therapy for clinically localized prostate cancer. JAMA 1998; 280:969-974.

[2] Meng MV, Elkin EP, Latini DM, Duchane J, Carroll PR, Treatment of patients with high risk localized prostate cancer. Results from cancer of the prostate strategic urological research endeavor (CaPSURE). J Urol 2005; 173:1557-1561.

[3] Yamamoto S, Kawakami S, Yonose J, et al. Long-term oncological outcome and risk stratification in men with high risk prostate cancer treated with radical prostatectomy. Jpn J Clin Oncol 2012; 42:541-547.

[4] American Joint Committee on cancer Prostate. Edge SB, Byrd DR, Compton CC, et al, AJCC cancer staging manual, 7th edn. Springer, New York, 2010: 457-468.

[5] Bolla M, Collette L, Blank L, et al. Long-term results with immediate androgen suppression and external irradiation in patients with locally advanced prostate cancer (an EORTC study):a phase 3 randomized trial. Lancet 2002; 360:103-106. 
Preoperative serum prostate-specific antigen level is a predictive factor for biochemical recurrence in high risk prostate cancer after radical prostatectomy

[6] Abdollah F, Sood A, Sammon JD, et al. Long-term cancer control outcomes in patients with clinically high-risk prostate cancer treated with robot-assisted radical prostatectomy: results from a multi-institutional study of 1100 patients. Eur Urol 2015; 68:497-505.

[7] Antunes AA, Leite KR, Dall'Oglio MF, Cury J, Srougi M, The effect of the number of biopsy cores on the concordance between prostate biopsy and prostatectomy gleason score; A prostate volume-controlled study. Arch Pathol lab Med 2008; 132:989-92.

[8] Van Poppel H, Joniau S. An analysis of radiacal prostatectomy in advanced stage and high-grade prostate cancer. Eur Urol 2008; 53:253-259.

[9] Hardeman SW, Causey JQ, Hickey DP, Soloway MS. Transrectal ultrasound for staging prior to radical prostatectomy. Urology $1989 ; 34: 175-80$.

[10] Augustin H, Fritz GA, Ehammer T, Auprich M, Pummer K.Accuracy of 3-Tesla magnetic resonance imaging for the stagingof prostate cancer in comparison to the Partin tables. Acta Radiol2009; 50:562-9.

[11] Ward JF, Slezak JM, Blute ML, Bergstralh EJ, Zincke H. Radical prostatectomy for clinical advanced (cT3) prostate cancer since the advent of prostate-specific antigen testing. BJU Int 2005; 95:751-756.

[12] Koie T, Mitsuzaka K, Yoneyama T, et al. Prostate-specific antigen density predicts extracapsular extension and increased risk of biochemical recurrence in patients with high-risk prostate cancer who underwent radical prostatectomy. Int J Clin Oncol 2015;20:176-181.

[13] Hamada R, Nakashima J, Ohori M, et al. Preoperative predictive factors and further risk stratification of biochemical recurrence in clinically localized high-risk prostate cancer. Int J Clin Oncol 2015 [Epub ahead of print] ;doi 10.1007/s10147-015-0923-3

[14] Matthews GJ, Motta J, Fracehia JA. The accuracy of transrectal ultrasound prostate volume estimation. J Clin Ultrasound 1996; 24:501-505.

[15] Lee JS, Chung BH. Transrectal ultrasound versus magnetic resonance imaging in the estimation of prostate volume as compared with radical prostatectomy specimens. Urol Int 2007; 78:323-327.

[16] Freeland SJ, Kane CJ, Presti Jr JC, et al. Comparison of preoperative prostate specific antigen density and prostate specific antigen for predicting recurrence after radical prostatectomy: Results from the serach data base. J Urol 2003; 169: 969-973.

[17] Aoun F, Albisinni S, Henriet B, Tombal B, Velthoven RV, Roumeguère T. Predictive factors associated with biochemical recurrence following radical prostatectomy for pathological T2 prostate cancer with negative surgical margins. Scand J Urol 2016; doi.10.1080/21681805. 2016.1263237

[18] Wagner M, Sokoloff M, Daneshmand S. The role of pelvic lymphadenoctomy for prostate cancer-therapeutic? J Urol 2008; 179:408-413.

[19] Suardi N, Dell'Oglio P, Gallina A, Briganti A.Evaluation of positive surgical margins in patients undergoing robot-assisted and open radical prostatectomy according to preoperative risk groups. Urol Oncol 2016; 34:57e1-57e7.

Citation: Tetsuya Shindo et al. Preoperative serum prostate-specific antigen level is a predictive factor for biochemical recurrence in high risk prostate cancer after radical prostatectomy.ARC Journal of Urology. 2017; 2(1):11-18.

Copyright: ( 2017 Dilip Tetsuya Shindo, et al. This is an open-access article distributed under the terms of the Creative Commons Attribution License, which permits unrestricted use, distribution, and reproduction in any medium, provided the original author and source are credited 